\title{
Reading through breakthroughs
}

\author{
The development of rechargeable batteries looks hugely successful on paper, but moving in leaps takes \\ fundamental breakthroughs, truly meaningful performance advances, and technological integration.
}

Breakthroughs in rechargeable batteries regularly make headlines these days. Some seem promising in the context of materials science and electrochemistry research in relation to discovery of an electrode, an electrolyte or another battery component; others strike us as larger-than-life claims, often lacking lucidity, with astonishing leaps forward in performance over conventional lithium-ion batteries. Regardless of potential issues of trustworthiness, the fact remains that conventional lithium-ion technologies are still dominant in the market.

A battery is a multi-component system. This means that one breakthrough in a single component is insufficient for real applications. Batteries operate in widely varying conditions, necessitating satisfactory performance in a string of metrics including energy and power density, cycling life, thermal stability, safety and cost, to name but a few.

Yet reported breakthroughs often occur in just one aspect of battery performance, making compromises in other metrics. Take a much-discussed report ${ }^{1}$ on the development of the aluminium-ion battery, for example. This battery makes use of an aluminium metal anode and a graphitic-foam cathode, both of which are inexpensive materials, and is shown to have an exceptionally high rate capability: it can be charged within one minute. However, its low capacity and energy density leave much room for improvement ${ }^{2}$.

Achieving high energy density is perhaps the main priority in today's battery research and development, as exemplified by the objective of the recently launched US Battery500 consortium to build a battery cell with a specific energy of $500 \mathrm{wh} \mathrm{kg} \mathrm{kg}^{-1}$, compared to the $\sim 200 \mathrm{wh} \mathrm{kg}^{-1}$ in today's electric vehicle batteries ${ }^{3}$. Using metallic lithium anodes has long been seen as an ideal approach to boost energy density; however, many lithium-dendrite-induced and solidelectrolyte-interface-induced issues such as parasitic reactions and short-circuiting hazards have hindered the application of lithium metal anodes. Writing in this issue (article no. 17083), Jian Xie and colleagues report a method to control dendrite growth by coating the separator with functionalized nanocarbon particles with immobilized lithium ions on their surfaces and, importantly, they demonstrate that this approach is not electrolyte-system specific. Nevertheless, while metal anode problems may be avoided, major breakthroughs are still needed in the search for appropriate cathode materials and cell architectures for practical rechargeable batteries ${ }^{4}$.

Single-aspect performance breakthroughs are plentiful in other promising advanced battery types. Published in this issue (article no. 17090), Jun Lu and colleagues report highly compact crystalline lithium sulfide nanoparticles, with a sulfur content as high as $88.5 \mathrm{wt} \%$, for lithium-sulfur batteries. High sulfur loading is essential to realise the high theoretical capacity of lithium-sulfur batteries. As such, their fabrication by burning lithium foils in a carbon disulfide vapour, as shown in this work, offers an intriguing idea for a scalable and inexpensive cathode design. However, although the researchers demonstrate high capacity and high energy density in their batteries, long-term cyclability at high rates needs to be improved.

Much of the research work in batteries is driven by performance improvement. This is perfectly understandable because the quality of the product is measured by its performance. However, breakthrough discoveries limited to single aspects of battery design cannot deliver a complete product in one step. In particular, for batteries that are already in the market or close to commercialization, true technological breakthroughs require improvements in all-round performance, not just single aspects. A particular example is the development of silicon-based batteries, requiring simultaneously meeting targets of cycling efficiency, stable solid-electrolyte interface and rate capability, to name but a few. This is why, although silicon-based batteries are already being implemented in some applications, only a small amount of silicon can be mixed with the graphite anodes and the full potential of silicon is yet to be exploited ${ }^{5}$.

Adding another layer of complexity when it comes to analysing breakthroughs, the question of whether they report truly meaningful performance metrics must be asked. One simple and typical example is a record-breaking claim on a specific capacity value based solely on active materials and a half-cell experiment. While the numbers may look unprecedented, they often bear little practical meaning when taking into account the whole cell weight or volume and a full-cell configuration. Other examples may not be as clear-cut. For instance, limiting depth-of-cycling (that is, restricting the full use of the available capacity in cycling) is an often-adopted practice in battery testing. However, caution must be taken with the obtained cyclability. To this end, Stephan Freunberger (article no. 17091) highlights the problems and difficulties with reporting true performance metrics. He emphasizes that details of cell components and operation parameters are essential to judge the performance metrics, and explains that high active material packing density and a small inactive-material-to-active-material ratio are key to achieving practically useful advances in battery performance. While these points are known to seasoned battery experts, the community is growing so fast that awareness should be raised in regard to what the meaningful performance metrics are, especially in research papers.

Finally, it is also important to encourage exploration and understanding of the materials and electrochemistry that underpin rechargeable batteries, because breakthroughs at the fundamental level offer hope for leaps forward in performance. In this respect, some advanced batteries such as lithium-air may not yet possess as satisfactory a performance as others do, but progress can be made through addressing major scientific challenges such as understanding the mechanisms of oxygen reduction at the cathode upon discharge (and its reverse reaction) and the mechanisms of cathode and electrolyte degradation. Simultaneously overcoming all scientific and technological challenges will not happen overnight and it will be both key breakthroughs and incremental steps - on fundamental and technological levels - that ultimately add up to real advances.

References

1. Lin, M.-C. et al. Nature 520, 324-328 (2015).

2. Highfield, V. Stanford University's aluminium-ion battery is a breakthrough, but it's not a big deal. alphr (8 April 2015).

3. White, F. Battery500 Consortium to Spark EV Innovations (Pacific Northwest National Laboratory, 2016).

4. Tikekar, M. D., Choudbury, S. Tu, Z. \& Archer, L. A. Nat. Energy 1, 16114 (2016).

5. Ruoff, C. Tesla tweaks its battery chemistry: a closer look at silicon anode development. Charged (23 September 2015). 